\title{
Circulatory markers of oxidative stress and dyslipidemia in male patients of chronic plaque psoriasis
}

Context: Psoriasis is one of the common chronic and recurrent inflammatory skin disorders. The inflammatory exudates in psoriasis are responsible for various lipid abnormalities as well as trigger a pro-oxidant and antioxidant imbalance resulting in copious generation of oxygen metabolites and proteases which may induce oxidative and proteolytic damage to plasma constituents and circulating red blood cells (RBCs) Aims: The aim was to evaluate dyslipidemia and erythrocyte oxidative stress as markers in plaque psoriasis. Materials and Methods: The study was performed on 120 male subjects, out of which 60 were patients of a moderate form of plaque psoriasis and 60 healthy age-matched controls. We evaluated lipid profile, RBC morphological indices such as total RBC count, hematocrit, hemoglobin concentration, hematimetric indices, osmotic fragility, and reticulocyte count. We also evaluated antioxidant defenses catalase, superoxide dismutase, glutathione peroxidase (GPX), and oxidant malondialdehyde (MDA) levels. Statistical Analysis Used: Independent sample $t$-test was used to compare the means between two groups. Results: Psoriasis patients showed a significant rise $(P<0.001)$ in total cholesterol, low-density lipoproteins (LDL), very LDL and triglycerides. The morphological indices of RBC presented with significantly reduced $(P<0.05)$ RBC count, $\mathrm{Hb}$ concentration and Hematocrit, significant increase $(P<0.05)$ in reticulocyte index and osmotic fragility. The antioxidant enzyme GPX activity was significantly lower while the oxidant MDA levels were significantly higher in patients with psoriasis $(P<0.001)$. Conclusions: Evaluation of the changes in RBC morphology, lipid profile, and antioxidant enzymes may be considered as biomarkers in predicting the severity of plaque psoriasis.

Key words: Antioxidant enzymes, lipid peroxidation, lipid profile, osmotic fragility, oxidative stress

Siddhartha Institute of Medical Sciences, and Research

Foundation, Gannavaram, Vijayawada, Andhra Pradesh, India

Address for the Correspondence:

Dr. Shruti Mohanty, Department of Biochemistry, Kamineni Institute of Medical Sciences, Narketpally, Nalgonda, Telangana, India.

E-mail: shruty.mohanty@ gmail.com

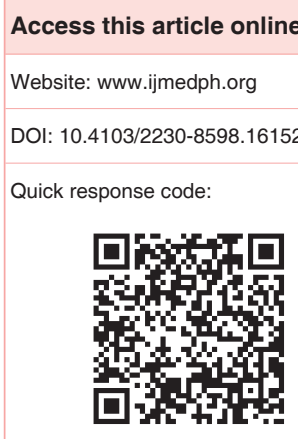

\section{INTRODUCTION}

Psoriasis is a chronic and recurrent T cell-mediated disease involving CD 4 and CD 8 lymphocytes. ${ }^{[1,2]}$ and a known oxidative stress condition. ${ }^{[3]}$ These cells, upon encounter with an antigen become activated and migrate to the skin where they release a plethora of cytokines including tumoral necrosis factor- $\infty$, Interferon- and interleukin-2, that initiate and perpetuate the inflammatory response in the skin. ${ }^{[3]}$ There are proliferation and accumulation of monocytes and macrophages in blood and tissues. ${ }^{[4]}$ The monocytes and macrophages release oxygen metabolites and proteases which cause oxidative and proteolytic damage to plasma constituents and red blood cells (RBCs). ${ }^{[5]}$ Psoriasis lesions that are clinically active, show infiltration of leukocytes, and several studies report high levels of leukocyte activation products in the peripheral blood of these patients. ${ }^{[3,4]}$ Activated white blood cells (WBCs) are important sources of reactive oxygen species (ROS) and by diffusing across the cell membrane, ROS may trigger an oxidative stress within the cells. ${ }^{[5]}$ ROS usually trigger an up-regulation of the antioxidant defenses, however, an oxidative stress will develop when an imbalance is developed between antioxidants and prooxidants. ${ }^{[6,7]}$ Human erythrocytes are important targets for the biological oxidative effects of free radicals as they are rich in polyunsaturated fatty acids, contain hemoglobin which is one of the most potent catalysts of lipid peroxidation. ${ }^{[8]}$ Therefore, the oxidative effects of oxygen free radicals on erythrocytes are greater than on other 
tissues. ${ }^{[9]}$ Presenting a limited biosynthesis capacity, the circulating erythrocyte suffers and accumulates physical and chemical changes, which become more pronounced with cell age, and whenever an unusual physical or chemical stress develops ${ }^{[8,9]}$ Many modifications are known to occur throughout the life span of the circulating RBCs, such as change in cell size, volume and in enzymatic activity. ${ }^{[8-10]}$ The invasion of the erythrocyte membrane by peroxidants, which occurs in inflammatory conditions like psoriasis, alters the cellular metabolic function and antioxidant defenses such as catalase (CAT), superoxide dismutase (SOD) and glutathione peroxidase (GPX). ${ }^{[11,12]}$ These components build up an efficient defense system inside the erythrocyte. SOD converts superoxide radicals into hydrogen peroxide, which generates the most reactive species, hydroxyl anion radical under the appropriate conditions. Hydrogen peroxide is degraded to water and oxygen via GPX at physiological concentrations. ${ }^{[12,13]}$ When hydrogen peroxide concentration increases until toxic levels, CAT also contributes to the metabolism of this substrate. In this way, they play an important role in the protective mechanisms against exposure to oxidative stress. By diffusing across the RBC membrane, oxygen metabolites trigger an oxidative stress within the cells which if assessed may prove to be an important biomarker of diagnosis and prognosis of the disease.

The present study focuses on the assessment of erythrocyte oxidative stress in patients with chronic plaque psoriasis by evaluating lipid peroxidation, RBC morphological changes and antioxidant defenses.

\section{MATERIALS AND METHODS}

The study comprised of total 120 male subjects aged 20-50 years, out of which 60 were patients diagnosed with moderate to severe form of plaque psoriasis in the outpatient Department of Dermatology, Kamineni Institute of Medical Sciences, Narketpally over a time period of 12.10.12-18.6.14. Patients were evaluated clinically by the dermatologist based on history, Cutaneous and systemic examination including location, severity of erythema, amount of scaling, and thickness of psoriatic plaques. 60 age-matched healthy controls were enrolled for this study, recruited from healthy volunteers and patients attending skin outpatient department for cosmetic problems like acne and pigment disturbances. The study was approved by Institutional Ethics Committee. Informed consents were taken from the patients as well as the controls, before collection of the blood sample.

\section{Inclusion criteria}

All the patients included were having a psoriasis area and severity index (PASI) score of 8-12 (PASI score 8-12 for moderate psoriasis) $\cdot{ }^{[14]}$

\section{Exclusion criteria}

1. The controls, as well as the patients, receiving any kind of medication, namely antioxidants, vitamins or methotrexate.
2. Patients with diabetes mellitus, renal diseases, hepatic diseases, and any other neuro-degenerative disorders.

3. Patients who had received any systemic or local steroid medication or any phototherapy treatment for at least 1 month prior to blood collection.

Clinical parameters were documented in a well-designed proforma prior to collection of $10 \mathrm{ml}$ of fasting venous blood from each of these patients, for carrying out hematological and biochemical investigations in central biochemistry laboratory of KIMS hospital.

\section{Erythrocyte morphology study}

Whole blood (ethylenediaminetetraacetic acid as anticoagulant) was used for determining RBC count; $\mathrm{Hb}$ concentration, $\mathrm{RBC}$ indices that is, mean corpuscular volume (MCV), mean corpuscular hemoglobin concentration $(\mathrm{MCH}), \mathrm{MCH}$ concentration $(\mathrm{MCHC})$, and reticulocyte index by an automated cell counter (Autocounter AC 970).

\section{Red blood cells osmotic fragility}

The osmotic fragility was measured by saline solutions buffered to $\mathrm{pH} 7.4$, used at different concentrations (1.0-9.0 g/l). Heparinized blood was added to these test tubes in the proportion of 1:100; Hemolysis was evaluated after $30 \mathrm{~min}$ of incubation at room temperature, by measuring the absorbance at $540 \mathrm{~nm}$ of the supernatant obtained after centrifugation (1500 $\mathrm{g}$ for $5 \mathrm{~min}$ ).

\section{Erythrocyte antioxidant defenses}

Heparinized blood was centrifuged at $1000 \mathrm{~g}$ for 10 minute at $4^{\circ} \mathrm{C}$, the buffy coat was discarded, and the isolated RBC pellet was hemolyzed in 4 times its volume of ice-cold high-performance liquid chromatography-grade water and again centrifuged at $4^{\circ} \mathrm{C}$. The erythrocyte lysate was then used to evaluate the CAT, SOD, and GPX activity.

\section{Glutathione peroxidase activity assay}

Glutathione peroxidase activity was determined with Cayman kits (Item no. 703102, Ann Arbor, MI 48108, USA) at $25^{\circ} \mathrm{C}$ by colorimetry at $340 \mathrm{~nm}$, based on the method of Paglia and Valentine, ${ }^{[15]}$ which requires cumene hydroperoxide as a substrate. Before analysis, the erythrocyte lysates were diluted to 20 -fold with sample buffer. The final concentration of reagents in the assay was those recommended by the manufacturer. The GPX activity was measured in $\mathrm{IU} / \mathrm{g}$ of $\mathrm{Hb}$.

\section{Superoxide dismutase activity assay}

Superoxide dismutase activity was determined with Cayman kits (item no. 706002 , Ann Arbor, MI 48108, USA) at $25^{\circ} \mathrm{C}$ by colorimetry at $340 \mathrm{~nm}$, based on the method of Marklund. ${ }^{[16]}$ This method employs xanthine and xanthine oxidase to generate superoxide radicals which react with 2-(-4-iodophenyl)-3-(4nitrophenol)-5-phenyl tetrazolium chloride to form red formazone dye. The SOD activity is then measured by the degree of inhibition 
of this reaction. SOD units were obtained from the standard curve expressed in $\mathrm{IU} / \mathrm{g}$ of $\mathrm{Hb}$.

\section{Catalase assay}

Catalase activity was assayed based on the method of Johansson and Borg, ${ }^{[17]}$ using the Cayman kits (item no. 707002, Ann Arbor, MI 48108 , USA). The method is based on the reaction of the enzyme with methanol in the presence of an optimal concentration of $\mathrm{H}_{2} \mathrm{O}_{2}$. The formaldehyde produced is measured calorimetrically with purpald as the chromogen at $340 \mathrm{~nm}$. CAT activity is calculated as the amount of enzyme that will cause the formation of $1.0 \mathrm{nmol}$ of formaldehyde per minute expressed in $\mathrm{nmol} / \mathrm{min} / \mathrm{ml}$.

\section{TBAR assay}

Plasma malondialdehyde (MDA) levels were determined by the method of Richard et al. ${ }^{[18]}$ using the Cayman kits (item no. 10009055 , Ann Arbor, MI 48108, USA). In this method after centrifuging the blood at $1000 \mathrm{~g}$ for $10 \mathrm{~min}$ at $4^{\circ} \mathrm{C}$, the top yellow plasma layer is pipetted off. The MDA-TBA adduct formed by the reaction of MDA and TBA under high temperature $\left(90-100^{\circ} \mathrm{C}\right)$ and acidic condition is measured colorimetrically at $530-540 \mathrm{~nm}$.

\section{Lipid profile}

Serum total cholesterol (TC), triglycerides (TG), high-density lipoprotein (HDL), low-density lipoprotein (LDL) were done by autoanalyzer (Hitachi 912). Very LDL (VLDL) was calculated by Friedewald's equation. ${ }^{[19]}$

\section{Body mass index}

The body mass index of all the subjects was calculated by the accepted formula weight $(\mathrm{kg}) /\left(\right.$ height $\left.[\text { meter }]^{2}\right)$.

\section{Statistical analysis}

The data were expressed as mean \pm standard deviation. Comparison of data was done by independent sample $t$-test and $P$ values were calculated using the Open EPI6 software (Open Epi Version 2.3.1 from Department of Epidemiology, Rollins School of Public Health, Emory University, Atlanta, GA 30322, USA). $P<0.05$ was considered to be significant.

\section{RESULTS}

All the 120 subjects were investigated for parameters of RBC morphological indices as well as antioxidant enzymes. The baseline data and lipid profile are described in Table 1. RBC indices are given in Table 2. The antioxidant defenses and MDA levels are compared. We observed significantly lower $(P<0.05)$ RBC count, $\mathrm{Hb}$ concentration and higher reticulocyte index, erythrocyte osmotic fragility in the psoriasis group $(P<0.05)$. There was no significant difference observed in $\mathrm{MCV}$ and $\mathrm{MCH}$, whereas MCHC of the psoriasis group was lower in comparison to control. The erythrocyte GPX activity was found to be significantly low $(P<0.05)$ and the plasma MDA levels were significantly higher in psoriasis patients in

\begin{tabular}{|c|c|c|c|}
\hline Parameters & $\begin{array}{l}\text { Controls } \\
(n=60)\end{array}$ & $\begin{array}{l}\text { Patients } \\
(n=60)\end{array}$ & $P$ \\
\hline Age (years) & $42.9 \pm 1.02$ & $43.2 \pm 1.64$ & $>0.05$ \\
\hline $\mathrm{BMI}\left(\mathrm{kg} / \mathrm{m}^{2}\right)$ & $19.72 \pm 1.87$ & $19.34 \pm 1.41$ & $>0.05$ \\
\hline TC (upto $200 \mathrm{mg} / \mathrm{dl}$ ) & $162.65 \pm 16$ & $198.72 \pm 23.45$ & $0.000^{* *}$ \\
\hline HDL (30-60 mg/dl) & $46.07 \pm 6.98$ & $44.64 \pm 3.56$ & $>0.05$ \\
\hline LDL (80-150 mg/dl) & $97.1 \pm 24.7$ & $132.59 \pm 26.58$ & $0.000^{* *}$ \\
\hline VLDL (10-30 mg/dl) & $22.73 \pm 5.72$ & $27.36 \pm 4.41$ & $0.000^{* *}$ \\
\hline $\begin{array}{l}\text { Triglycerides (upto } \\
150 \mathrm{mg} / \mathrm{dl} \text { ) }\end{array}$ & $115.04 \pm 26.57$ & $138.29 \pm 34.76$ & $0.000^{* *}$ \\
\hline
\end{tabular}

$* * P<0.001 . \mathrm{BMI}=$ Body mass index, $\mathrm{HDL}=$ High-density lipoprotein, $\mathrm{LDL}=\mathrm{Low}-$ density lipoproteins, VLDL = Very low-density lipoproteins, $T C=$ Total cholesterol

\begin{tabular}{|c|c|c|c|}
\hline Parameters & $\begin{array}{l}\text { Controls } \\
(n=60)\end{array}$ & $\begin{array}{l}\text { Patients } \\
(n=60)\end{array}$ & $P$ \\
\hline RBCs $\left(5-6 \times 10^{12} / \mathrm{L}\right)$ & $5.26 \pm 0.53$ & $5.01 \pm 0.39$ & $0.003^{*}$ \\
\hline $\mathrm{Hb}(14-18 \mathrm{~g} \%)$ & $15.41 \pm 1.5$ & $14.18 \pm 1.02$ & $0.02^{*}$ \\
\hline Reticulocyte index (<1\%) & $1.10 \pm 0.08$ & $1.33 \pm 0.61$ & $0.004^{*}$ \\
\hline PCV (40-50 vol \%) & $43.6 \pm 1.83$ & $42.8 \pm 1.52$ & $0.01^{*}$ \\
\hline MCV (78-94 fl) & $84.72 \pm 5.34$ & $83.3 \pm 4.38$ & $>0.05$ \\
\hline $\mathrm{MCHC}(32-38 \%)$ & $32.5 \pm 2.61$ & $31.6 \pm 1.42$ & $0.02^{*}$ \\
\hline $\mathrm{MCH}(28-30 \mathrm{pg})$ & $28.25 \pm 5.34$ & $27.2 \pm 0.21$ & $>0.05$ \\
\hline $\begin{array}{l}\text { Osmotic fragility } \\
(0.48-0.34 \% \text { of } \mathrm{NaCl} \\
\text { solution })\end{array}$ & $0.42 \pm 0.02$ & $0.44 \pm 0.03$ & $0.000^{* *}$ \\
\hline CAT ( $n$ moles $/ \mathrm{min} / \mathrm{ml})$ & $552.85 \pm 77.25$ & $535 \pm 73.8$ & $>0.05$ \\
\hline SOD (IU/g of Hb) & $1612 \pm 252$ & $1568.06 \pm 132.7$ & $>0.05$ \\
\hline GPX (IU/g of $\mathrm{Hb})$ & $51 \pm 9.6$ & $47.6 \pm 8.2$ & $0.039^{*}$ \\
\hline $\operatorname{MDA}(\mathrm{nmol} / \mathrm{dl})$ & $2.4 \pm 0.73$ & $6.72 \pm 0.89$ & $0.000^{* *}$ \\
\hline
\end{tabular}

Data expressed as mean \pm SD. $* P<0.05, * * P<0.001$. RBC $=$ Red blood cells, $\mathrm{MCV}=$ Mean corpuscular volume, $\mathrm{MCHC}=$ Mean corpuscular hemoglobin concentration, CAT = Catalase, SOD = Superoxide dismutase, GPX = Glutathione peroxidase, $\mathrm{MDA}=$ Malondialdehyde, $\mathrm{MCH}$ : Mean corpuscular hemoglobin, $\mathrm{Hb}=$ Hemoglobin, SD = Standard deviation, $\mathrm{PCV}=$ Packed cell volume

comparison to the controls $(P<0.001)$. There was no significant difference observed in CAT and SOD activity among both the groups.

\section{DISCUSSION}

Plaque psoriasis is one of the most common forms of psoriasis. This inflammatory skin disease often encountered clinically seems to impose an oxidative stress condition. ${ }^{[2]}$ Previous studies have reported dyslipidemia to be significantly associated with psoriasis. ${ }^{[20,21]}$ In the current study, we observed significantly high levels of TC, LDL, VLDL, and TG in psoriasis patients than controls. However, we did not observe any change in the levels of HDL. Our study is concurrent with that of Lateef et al., who has reported similar findings in their study on lipid profile and protein fractions in psoriasis. ${ }^{[2]}$ The association of dyslipidemia may be due to chronic inflammation, which is a characteristic feature of psoriasis, plays a role in the initiation and progression 
of dyslipidemia and atherosclerosis. ${ }^{[20-22]}$ In the present study, We found that psoriasis patients presented significantly higher number of WBCs [Figure 1]. The leukocytosis is mainly due to an increased number of neutrophils.

It has been observed that psoriasis is characterized by high levels of circulating neutrophil activation products, that is, elastase and lactoferrin..$^{[5]}$ The higher plasma levels of elastase and lactoferrin may be responsible for further neutrophil activation. ${ }^{[4,5]}$ It is known that neutrophil activation is also linked to the production of oxygen metabolites, which may induce oxidative and proteolytic modifications to plasma constituents and neighboring cells such as circulating erythrocytes. ${ }^{[12]}$ The oxidative stress is bestmanifested on erythrocyte, as the RBC membrane is rich in lipids, does not have a nucleus, all the metabolic enzymes are present in its cytoplasm. ${ }^{[10]}$ Hence, the enzyme activity can be studied and correlated with the extent of damage done by an inflammatory reaction. Some oxygen metabolites are highly reactive, whereas others, like hydrogen peroxide, are less reactive and have the ability to diffuse easily across the erythrocyte membranes. ${ }^{[3]}$ We observed a significant increase in the osmotic fragility and reticulocyte index in moderate form of plaque psoriasis $(P<0.05)$ [Table 1]. The ROS may, therefore, impose oxidative modifications upon the membrane and within the RBC, leading to structural modifications which may cause premature senescence and hemolysis. ${ }^{[23,24]}$ This may be the reason for a significant rise in osmotic fragility, reticulocyte index, fall in MCHC and hematocrit $(P<0.05)$ observed in our study. Except MCHC, other RBC indices like MCV, MCH were not altered significantly in the present study. Our results are similar to the findings of Rocha-Periera et al. who found that the blood indices were markedly altered in severe form of psoriasis but not in mild or moderate forms. ${ }^{[1,2]}$

The evaluation of plasma levels of lipid peroxidation [Table 2] showed a significantly higher value of MDA levels in psoriasis $(P<0.001)$ indicating higher lipid peroxidation. The ROS causes peroxidation of $\mathrm{RBC}$ membrane, the peroxidants attach to the

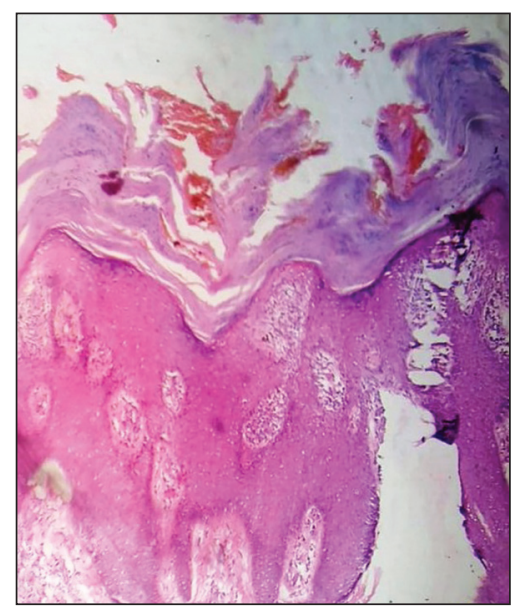

Figure 1: Histopathology of plaque psoriasis demonstrating neutrophilic infiltration of the dermis
RBC membrane and trigger alteration of the cellular antioxidant enzyme activity. ${ }^{[2]}$ Gaining the inside of the RBC, they will trigger the erythrocyte antioxidant defense mechanisms. ${ }^{[25]}$ In psoriasis patients, we observed a significant reduction in the erythrocyte antioxidant enzymatic activity GPX $(P<0.05)$. This enzyme is particularly involved in the detoxification of the cell in oxygen radicals. Our study is concurrent with that of Santosh-Silvia et al. who also had reported similar findings. ${ }^{[18]}$ Surprisingly, we did not observe any significant difference in the CAT as well as SOD activity in psoriasis patients. Our study does not agree with Therond et al. and Rocha-Pereira et al. who has reported significantly lower activities of CAT in psoriasis patients. ${ }^{[26,1]}$ In the case of SOD, some authors reported a reduced activity in psoriasis patients while others, including ourselves, found no significant change. ${ }^{[26,27]}$ This may be due to other associated factors like dietary patterns, lifestyle, exercise etc., which may interfere with the antioxidant status of the body. ${ }^{[27]}$ Furthermore, the difference may be due to the fact that patients included in previous studies, suffer from different forms of psoriasis such as localized plaque psoriasis, pustular psoriasis or erythroderma and they may be undergoing various forms of treatments, but our patients had no erythroderma or generalized pustulosis. Our findings are in concurrent with that of Rocha-Pereira et al. who reported decrease in GPX activity in mild and moderate form of psoriasis, followed by marked decrease in activity of all the three enzymes, as the disease progresses from mild to severe form with enlargement of the psoriatic lesion..$^{[1,4]}$

In summary, we found erythrocyte morphological changes denoting enhanced damage, namely a rise in osmotic fragility, reticulocyte index, oxidant and antioxidant enzyme activity can be associated with progress of the disease. Reductions in total RBC count, rise of reticulocyte index, plasma MDA levels with failure of membrane antioxidant defenses, and a rise in the osmotic fragility, are all markers of RBC senescence or damage, and these changes were observed in psoriasis. Since the present study focuses on the erythrocyte as a marker for the progress of the disease, we suggest that a regular evaluation of these RBC markers in patients with moderate plaque psoriasis could be of value in predicting in advance the worsening of the disease and therefore enabling earliest and better intervention with antioxidants as adjunctive therapy.

However, further research should be carried out on other inflammatory and stress markers like C-reactive protein, ferritin, and ceruloplasmin to augment the present study.

\section{REFERENCES}

1. Rocha-Pereira P, Santos-Silva A, Rebelo I, Figneiredo A, Quintanilha A, Teixeira F. Erythrocyte damage in mild and severe psoriasis. $\mathrm{Br} \mathrm{J}$ Dermatol 2004;150:232-44.

2. Rocha-Pereira P, Santos-Silva A, Rebelo I, Figueiredo A, Quintanilha A, Teixeira F. Dislipidemia and oxidative stress in mild and in severe psoriasis as a risk for cardiovascular disease. Clin Chim Acta 2001;303:33-9.

3. Kökçam I, Naziroglu M. Antioxidants and lipid peroxidation status in the blood of patients with psoriasis. Clin Chim Acta 1999;289:23-31. 
4. Rocha pereira P, Rebelo I, Santosh-Silva A, Figueitedo A, Ferra MA, Quintanilha A, et al. Leukocyte activation and oxidative stress inpsoriasis. Br J Pharmacol 1999;127:79-83.

5. Orem A, Deger O, Cimsit G, Bahadir S. Plasma polymorphonuclear leukocyte elastase levels and its relation to disease activity in psoriasis. Clin Chim Acta 1997;264:49-56.

6. Weiss SJ. Neutrophil-mediated methemoglobin formation in the erythrocyte. The role of superoxide and hydrogen peroxide. J Biol Chem 1982;257:2947-53.

7. Claster S, Chiu DT, Quintanilha A, Lubin B. Neutrophils mediate lipid peroxidation in human red cells. Blood 1984;64:1079-84.

8. Asgary S, Naderi G, Ghannady A. Effects of cigarette smoke, nicotine and cotinine on red blood cell hemolysis and their - SH capacity. Exp Clin Cardiol 2005;10:116-9.

9. Ugbebor OU, Oseni BA, Arinolas OG, Osazuwa F, Zabayo OJ. Red blood cell susceptibility to oxidants in chronic cigarette smokers. Res J Pharm Biol Chem Sci 2011;2:380-8.

10. Gutteridge JM. Lipid peroxidation and antioxidants as biomarkers of tissue damage. Clin Chem 1995;41:1819-28.

11. Pastore A, Federici G, Bertini E, Piemonte F. Analysis of glutathione: Implication in redox and detoxification. Clin Chim Acta 2003;333:19-39.

12. Santos-Silva A, Rebelo MI, Castro EM, Belo L, Guerra A, Rego C, et al. Leukocyte activation, erythrocyte damage, lipid profile and oxidative stress imposed by high competition physical exercise in adolescents. Clin Chim Acta 2001;306:119-26.

13. Griffiths CE, Barker JN. Psoriasis. In: Champion RH, Burton JL, Burns DA, Breathnach SM, editors. Text Book of Dermatology. $8^{\text {th }}$ ed. Oxford: Black Well Sciences; 1998. p. 20.1-20.14

14. Zangrilla A, Talamonti M, Saraceno R, Chimenti S. Clinical severity instruments. In: Sergio Chimenti, editor. Psoriasis. $1^{\text {st }}$ ed. New Delhi: SeeFirenze, Viva Books; 2010. p. 115-7.

15. Paglia DE, Valentine WN. Studies on the quantitative and qualitative characterization of erythrocyte glutathione peroxidase. J Lab Clin Med 1967;70:158-69.

16. Marklund S. Distribution of CuZn superoxide dismutase and $\mathrm{Mn}$ superoxide dismutase in human tissues and extracellular fluids. Acta Physiol Scand Suppl 1980;492:19-23.

17. Johansson LH, Borg LA. A spectrophotometric method for determination of catalase activity in small tissue samples. Anal Biochem 1988;174:331-6.

18. Richard MJ, Portal B, Meo J, Coudray C, Hadjian A, Favier A. Malondialdehyde kit evaluated for determining plasma and lipoprotein fractions that react with thiobarbituric acid. Clin Chem 1992;38:704-9.

19. Friedewald WT, Levy RI, Fredrickson DS. Estimation of the concentration of low-density lipoprotein cholesterol in plasma, without use of the preparative ultracentrifuge. Clin Chem 1972;18:499-502.

20. Zari J, Tayyebi Meibodi N, Yalda N. Serum lipids abnormalities and Psoriasis. Indian J Dermatol 2007;52:89-92.

21. Weinstein GD, Frost P. Abnormal cell proliferation in psoriasis. J Invest Dermatol 1968;50:254-9.

22. Lateef A, Mohanty S, Reddy D, Rao P. Atherogenic index and proteinfractions in Psoriasis. Int J Med Pubic Health 2011;1:25-30.

23. Drewa G, Malinowska EK, WoźniakA. Activity of superoxide dismutaseand catalase and the level of lipid peroxidation products reactive with TBA in patients withpsoriasis. Med Sci Monit 2002;8:338-43.

24. Placer ZA, Cushman LL, Johnson BC. Estimation of product of lipid peroxidation (malonyl dialdehyde) in biochemical systems. Anal Biochem 1966;16:359-64.

25. Matkovics B, Szabo L, Varga IS. Determination of enzyme activities in lipid peroxidation andglutathione pathways. Laboratory Diagnostics 1988;15:248-9.

26. Thérond P, Gerbaud P, Dimon S, Anderson WB, Evain-Broin D, Raynaud F Antioxidant enzymes in psoriatic fibroblasts and erythrocytes. J Invest Dermatol 1996;106:1325-8.

27. Naldi L, Parazzini F, Peli L, Chatenoud L, Cainelli T. Dietary factors and the risk of psoriasis. Results of an Italian case-control study. Br J Dermatol 1996;134:101-6.

How to cite this article: Metta S, Kumar MA, Basalingappa RD, Uppala S, Mohanty S. Circulatory markers of oxidative stress and dyslipidemia in male patients of chronic plaque psoriasis. Int J Med Public Health 2015;5:208-12.

Source of Support: Kamineni Education Society, Conflicts of Interest: None declared. 